\title{
PREVALENCE OF FELINE CORONAVIRUS IN CATS OF AN ANIMAL SHELTER IN LATVIA
}

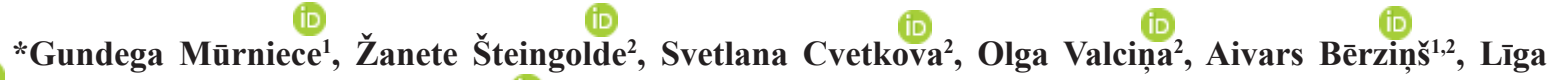 \\ Kovaḷčuka', Kaspars Kovaḷenko' ${ }^{1}$ (D) \\ ${ }^{1}$ Latvia University of Life Sciences and Technologies, Latvia \\ ${ }^{2}$ Institute of Food Safety, Animal Health and Environment "BIOR", Latvia \\ *Corresponding author's email: gundega.murniece@gmail.com
}

\begin{abstract}
Feline coronavirus $(\mathrm{FCoV})$ is ubiquitous in the domestic cat (Felis catus) population. The aim of this study was to determine the prevalence and potential predisposing factors of FCoV in cats of an animal shelter in Latvia and to compare the prevalence between cats in the quarantine area and resident cats in the adoption area. Oropharyngeal and faecal swabs and blood samples were collected from 40 domestic shorthair cats from an animal shelter in Jelgava, Latvia. Swabs were analyzed for FCoV RNA by reverse transcriptase-polymerase chain reaction (RT-PCR). Blood serum samples were tested for FCoV specific antibodies by indirect enzyme-linked immunosorbent assay (ELISA). FCoV RT-PCR positivity in oropharyngeal and rectal swabs was 7.5\% (3/40) and 72.5\% (29/40), respectively. Additionally, FCoV seroprevalence was $67.5 \%$ (27/40). The proportion of cats shedding FCoV within the adoption $(72.7 \%)$ and quarantine $(72.2 \%)$ areas was similar $(\mathrm{p}=0.55)$. The prevalence of FCoV faecal shedding in young cats was significantly higher $(\mathrm{p}<0.05)$ than in adult cats. Sex had no significant effect on FCoV RT-PCR positivity. Further studies on larger cat population including different population types are needed to determine the overall prevalence and epidemiological patterns of FCoV infection in Latvia.

Key words: coronavirus $(\mathrm{CoV})$, feline infectious peritonitis (FIP), feline coronavirus (FCoV), cats, animal shelter, RT-PCR.
\end{abstract}

\section{Introduction}

Coronaviruses (CoVs) are enveloped viruses with an exceptionally large $(27-32 \mathrm{~kb})$ positive-stranded RNA genome. They are divided into four different genera: alpha, beta, gamma, and delta coronaviruses based on their genomic characteristics (Le Poder, 2011). CoVs are a diverse group of viruses that are common in nearly every species of mammals and birds (Pedersen, 2014). Because of their large RNA genome, CoVs have moderate to high mutation and recombination rate, and this facilitates successful cross-species transmission ( $\mathrm{Su}$ et al., 2016). This paper focuses on feline coronavirus (FCoV) which is the most common pathogen identified in the faeces of cats (Felis catus) and is the causative agent of one of the most researched infectious diseases of cats: feline infectious peritonitis (FIP) (Pedersen, 2014). Feline coronaviruses (FCoVs) belong to the genus Alphacoronavirus and are grouped into two biotypes: low virulent $\mathrm{FCoV}$ which is primarily a pathogen of the gastrointestinal tract and highly virulent $\mathrm{FCoV}$ which has arisen from spontaneous mutations in the low virulent FCoV. Highly virulent FCoV has gained tropism for monocytes/macrophages enabling systemic spread (O’Brien et al., 2018) and a subset of infections (7-14\%) with highly virulent FCoV result in almost uniformly fatal FIP (Addie et al., 2020). Although highly virulent FCoV is responsible for the development of FIP, low virulent FCoV is the real culprit behind the maintenance of $\mathrm{FCoV}$ in multi-cat households, meaning that persistently infected and shedding cats play a key role in the epidemiology of FIP (Kipar \& Meli, 2014). Once a cat has been infected with low virulent $\mathrm{FCoV}$, there is a potential for FIP to develop. Some strains are more prone to cause FIP than others. FCoV is endemic in the domestic cat population worldwide, especially within multi-cat households and catteries with seropositivity rates approaching 90\% (Tekes $\&$ Thiel, 2016). There is currently no approved treatment for FIP and no effective vaccine against FCoV (O'Brien et al., 2018); therefore, detection and removal of FCoV shedders, as well as good hygiene practices are the essential tools for preventing transmission of FCoV. The importance of FCoV is not limited to the cat population only, as cats live in close contact with humans and are the most frequently kept species of companion animals (106 million) in Europe (FEDIAF, 2019). Knowing the high densities of domestic cats, the high prevalence of $\mathrm{FCoV}$ and the fact that $\mathrm{CoVs}$ are continuously adapting themselves to new hosts, FCoV cannot be neglected in terms of the One Health concept. There is considerable knowledge on the prevalence of FCoV and associated risk factors in many countries, but at the present time information on the current burden of FCoV in shelters in Latvia has not been examined; however, epidemiological results can differ among geographic locations (Worthing et al., 2012). The aim of the present study was to estimate the prevalence and potential predisposing factors of $\mathrm{FCoV}$ in cats of an animal shelter in Latvia and to compare the prevalence between cats in the quarantine area and resident cats in the adoption area. 


\section{Materials and Methods}

\section{Study population}

Samples from 40 domestic shorthair cats (22 male and 18 female) were collected from an animal shelter in Jelgava, Latvia, between September 2020 and January 2021. Examined cats were housed in a quarantine zone for incoming cats and in an area designated for resident cats. Age was provided by the owners who surrendered their cats or estimated by shelter staff in stray cats. The youngest cat tested was 2 month old, but the oldest was 12 years old; they were categorized as juvenile $(<1$ year) and adults $(\geq 1$ year). The clinical condition of all cats enrolled in this study was not an excluding factor. The study was approved by the Committee for the Protection of Animals Used for Scientific Purposes of the Food and Veterinary Service of the Republic of Latvia (certificate of approval No 119).

Sample collection

From each cat two polyester-tipped swabs (oropharyngeal and rectal) and one blood sample were collected. No anesthetic when collecting the samples was used, only proper handling, and a minimum of physical restraint. For swab collection, transport, and maintenance UTM ${ }^{\circledR}$ paired with COPAN FLOQ Swabs ${ }^{\circledR}$ were used. Commercial UTM $^{\mathrm{TM}}$ conical tubes were filled with $3 \mathrm{~mL}$ UTM $^{\mathrm{TM}}$ medium. Half of the viral transport medium was poured into sterile 1.5 $\mathrm{mL}$ Eppendorf Tubes ${ }^{\circledR}$ before taking the swabs. For the collection of oropharyngeal specimen, the minitip size swab was inserted into the caudal oropharynx and tonsillar areas. The sample was collected by rubbing the polyester-tipped shaft against the caudal oropharynx while trying to avoid contact with the tongue, teeth, and gums. Then the swab was inserted into the $\mathrm{UTM}^{\mathrm{TM}}$ tube until the breakpoint was level with the tube opening and the swab shaft was broken off at the breaking point. The second regular size swab was inserted $1.0-1.5 \mathrm{~cm}$ into the rectum. The sample was collected by gently rolling the swab against the rectal mucosa, then the swab was inserted in the prefilled Eppendorf Tube ${ }^{\circledR}$ and the shaft was cut leaving a tip of the swab into viral transport medium. For blood collection vacuum tube with clot activator and 23 -gauge butterfly catheter were used. The cephalic vein was occluded with a tourniquet, the venipuncture site was clipped and cleaned with $70 \%$ isopropyl alcohol pad. Subsequently, the butterfly catheter was inserted intravenously. From each cat about 0.7 to $1.5 \mathrm{~mL}$ of peripheral blood was obtained. Then the tourniquet was released, the catheter was withdrawn and pressure to the puncture site was applied.

\section{Serological testing}

All blood serum samples were tested for the presence of specific antibodies to $\mathrm{FCoV}$ by an indirect enzyme-linked immunosorbent assay (ELISA) using a commercial INGEZIM CORONA FELINO indirect
ELISA kit (Ingenasa, Spain). The procedure of the test was carried out according to the manufacturer's instructions. Briefly, a commercial plate is already coated with the specific FCoV antigen. For the reaction $200 \mu \mathrm{L}$ of serum dilution $1 / 200$ were added to the plate and incubated. If the samples contained specific antibodies to $\mathrm{FCoV}$, they bound to the antigen. The plate was washed and the specific peroxidase conjugate was added. The second washing of the plate followed and the substrate was added to the wells. The Multiskan FC ${ }^{\circledR}$ spectrophotometer (Life Technologies, Singapore) was used to measure the optical density (OD) of the colorimetric reaction. The cut-off value was determined according to the user manual of the kit. The samples with an OD value higher than the cut-off were considered positive and having specific antibodies to $\mathrm{FCoV}$. The samples with an $\mathrm{OD}$ value lower than the cut-off were considered as negative for the presence of specific antibodies to $\mathrm{FCoV}$.

Detection of FCoV by reverse transcriptasepolymerase chain reaction (RT-PCR)

Viral RNA was extracted from $200 \mu \mathrm{L}$ of sample using the IndiSpinPathogen kit (INDICAL, Leipzig, Germany). The RNA was eluted in $100 \mu \mathrm{L}$ of elution buffer and stored at $-80{ }^{\circ} \mathrm{C}$. CoV screening was performed by a pan-coronavirus one-step RT-PCR followed by sequencing of the amplified product (440 bp) to confirm CoV identification. PCR was performed by adding $5 \mu \mathrm{L}$ of extracted RNA to $20 \mu \mathrm{L}$ of the SuperScript ${ }^{\mathrm{TM}}$ III One-Step RT-PCR System with Platinum ${ }^{\mathrm{TM}} \mathrm{Taq}$ DNA polymerase kit (Invitrogen, Carlsbad, USA) reaction mixture containing $0.5 \mu \mathrm{M}$ of each primer (RdRP2-F GGTTGGGACTATCCTAAGTGTGA and RdRP2-R CCATCATCAGATAGAATCATCATA) (Poon et al., 2005). RT-PCR was carried out at $50{ }^{\circ} \mathrm{C}$ for $30 \mathrm{~min}$, followed by the activation of the DNA polymerase at $95^{\circ} \mathrm{C}$ for $2 \mathrm{~min}$, and by 40 cycles in three steps: $95^{\circ} \mathrm{C}$ for $30 \mathrm{~s}, 50{ }^{\circ} \mathrm{C}$ for $30 \mathrm{~s}$, and $68^{\circ} \mathrm{C}$ for $1 \mathrm{~min}$. An additional extension for $10 \mathrm{~min}$ at $68{ }^{\circ} \mathrm{C}$ was added at the end of the run. The RdRp PCR products were enzymatically purified using ExoI and FastAP (Life Technologies, Fermentas, Lithuania) and were subjected to nucleotide sequence analysis.

\section{Statistical methods}

Descriptive statistics for the age of the cat, sex, area in the shelter and $\mathrm{FCoV}$ status in all sample types was calculated using Student's t-tests for unpaired samples. P-values of less than 0.05 were considered statistically significant. Additionally, the Pearson correlation coefficient was calculated for RT-PCR and ELISA results.

\section{Results and Discussion}

A total of 40 cats from a single shelter were included in the study, of those $22(55 \%)$ resided in the 
Table 1

RT-PCR and indirect ELISA results according to animal sex and age

\begin{tabular}{|l|l|c|c|c|}
\hline \multirow{2}{*}{ Factor } & \multirow{2}{*}{\begin{tabular}{l} 
Category \\
\cline { 3 - 5 }
\end{tabular}} & $\begin{array}{c}|c| \\
\text { rectal swabs } \\
\text { (FCoV RNA) } \\
\text { (No. Positive/Total samples) }\end{array}$ & $\begin{array}{c}\text { RT-PCR } \\
\text { oropharyngeal swabs } \\
\text { (FCoV RNA) }\end{array}$ & $\begin{array}{c}\text { Indirect ELISA } \\
\text { sorum samples } \\
\text { (FCoV specific antibodies) } \\
\text { (No. Positive/Total samples) }\end{array}$ \\
\hline \multirow{2}{*}{ Sex } & Male & $16 / 22$ & $1 / 22$ & $12 / 22$ \\
\cline { 2 - 5 } & Female & $13 / 18$ & $2 / 18$ & $15 / 18$ \\
\hline Age & $<1$ year & $13 / 14$ & $0 / 14$ & $9 / 14$ \\
\cline { 2 - 5 } & $\geq 1$ year & $16 / 26$ & $3 / 26$ & $18 / 26$ \\
\hline
\end{tabular}

adoption area and 18 (45\%) were kept in quarantine. The overall prevalence of previous or ongoing $\mathrm{FCoV}$ infection was $82.5 \%$ (33/40). Out of the 40 cats, 29 cats $(73 \%)$ were shedding $\mathrm{FCoV}$, but 27 cats $(68 \%)$ had anti-FCoV antibodies. There was no significant difference $(\mathrm{p}=0.55)$ between the proportion of cats shedding FCoV within the adoption (72.7\%) and quarantine $(72.2 \%)$ areas. These results demonstrate that compartmentalization of the particular shelter into individual sections did not reduce transmission of $\mathrm{FCoV}$ and different prevention strategies for $\mathrm{FCoV}$ infection should be applied. Shedding cats are responsible for the persistent presence of $\mathrm{FCoV}$ in the domestic cat population (Felten et al., 2020) therefore, in order to avoid continual reinfection, shedders must be isolated. In previous studies estimated FCoV seroprevalence in multicat environment was highly variable, ranging from $25.6 \%$ in the United Kingdom (Cave et al., 2004) to $82 \%$ in Italy (Pratelli, 2008) depending on various factors such as population density, husbandry practices, time spent in the shelter before sampling, age, breed, and health status (Cave et al., 2004). Faecal shedding, as determined by RT-PCR from rectal swabs, in previous studies with mixed-breed cats in multicat environment was similar to our results. The overall prevalence of $\mathrm{FCoV}$ infection in the cat population in Malaysia and Germany was 70\% (Sharif et al., 2009) and 76.5\% (Klein-Richers et al., 2020), respectively.

The whole study population consisted of $22(55 \%)$ neutered males and $18(45 \%)$ neutered females. We found no significant correlation between FCoVpositivity and the sex of the cats $(\mathrm{p}=0.55)$. FCoV prevalence in male cats $(72.7 \%)$ was almost the same as in female cats $(72.2 \%)$. Among the $29 \mathrm{FCoV}$ positive cats, $13(45 \%)$ were less than 1 year old and $16(55 \%)$ were older than 1 year. Prevalence of FCoV fecal shedding in young cats $(92.9 \%)$ was significantly higher $(\mathrm{p}<0.05)$ than in adult cats $(61.5 \%)$ (Table 1$)$. We observed that young age was the only significant factor associated with FCoV shedding (RT-PCR positivity in rectal swabs). Our findings support previous studies that found a significant correlation between FCoV-positivity and the young age but no significant association between FCoV-positivity and sex of the cats (Klein-Richers et al., 2020; Pedersen, Allen, \& Lyons, 2008).

FCoV RNA was detected in $3 / 40$ (7.5\%) oropharyngeal swabs and in $29 / 40(72.5 \%)$ rectal swabs but FCoV specific antibodies in 27/40 (67.5\%) blood serum samples. The proportion of positive rectal swabs potentially could be even higher because only a single rectal swab from each cat was analyzed and according to previous studies, $70-80 \%$ of infected cats are intermittent shedders (Klein-Richers et al., 2020). Overall, $85 \%$ (23/27) of FCoV specific antibodypositive cats shed $\mathrm{FCoV}$ in their faeces. There was a moderate positive correlation between the presence of FCoV specific antibodies and FCoV shedding in feces $(\mathrm{r}=0.41, \mathrm{p}<0.01)$. This observation is in accordance with previous reports which showed that cats with antibodies were more likely to be the virus shedders than non-shedders (Felten et al., 2020; Pedersen, Allen, \& Lyons, 2008).

However, the prevalence of $\mathrm{FCoV}$ in shelter cats cannot be extrapolated to the overall cat population of Latvia. Further studies on larger cat population including different population types are needed to determine the overall prevalence and epidemiological patterns of $\mathrm{FCoV}$ in Latvia.

\section{Conclusions}

1. In this study, the prevalence of previous or ongoing FCoV infection in cats of an animal shelter in Latvia was $82.5 \%$; additionally, young age was a predisposing factor for $\mathrm{FCoV}$ infection.

2. The difference between cats shedding FCoV within the adoption (72.7\%) and quarantine (72.2\%) areas was not significant $(\mathrm{p}=0.55)$, thereby new prevention strategies should be applied to reduce FCoV infection and control FIP outbreaks since compartmentalization did not decrease the spread of $\mathrm{FCoV}$. 
3. A follow-up study should be performed to investigate the development of FIP in conjunction with RNA sequencing of FCoVs to identify specific mutations in the $\mathrm{FCoV}$ genome.

\section{Acknowledgements}

Research was funded by the State Research Programme 'Covid-19 mitigation' project 'Multidisciplinary approach to monitor, mitigate and contain COVID-19 and other future epidemics in Latvia' (No.VPP-COVID-2020/1-0008).

\section{References}

Addie, D.D., Curran, S., Bellini, F., Crowe, B., Sheehan, E., Ukrainchuk, L., \& Decaro, N. (2020). Oral Mutian ${ }^{\circledR X}$ stopped faecal feline coronavirus shedding by naturally infected cats. Research in Veterinary Science. 130, 222-229. DOI: 10.1016/j.rvsc.2020.02.012.

Cave, T.A., Golder, M.C., Simpson, J., \& Addie, D.D. (2004). Risk factors for feline coronavirus seropositivity in cats relinquished to a UK rescue charity. Journal of Feline Medicine and Surgery. 6(2), 53-58. DOI: 10.1016/j.jfms.2004.01.003.

FEDIAF. (2020). European Facts \& Figures 2019. Brussels: The European Pet Food Industry.

Felten, S., Klein-Richers, U., Hofmann-Lehmann, R., Bergmann, M., Unterer, S., Leutenegger, C.M., \& Hartmann, K. (2020). Correlation of Feline Coronavirus Shedding in Feces with Coronavirus Antibody Titer. Pathogens. 9(8), 598. DOI: 10.3390/pathogens9080598.

Kipar, A., \& Meli, M.L. (2014). Feline Infectious Peritonitis. Veterinary Pathology. 51(2), 505-526. DOI: $10.1177 / 0300985814522077$.

Klein-Richers, U., Hartmann, K., Hofmann-Lehmann, R., Unterer, S., Bergmann, M., Rieger, A., ... Felten, S. (2020). Prevalence of Feline Coronavirus Shedding in German Catteries and Associated Risk Factors. Viruses. 12(9), 1000. DOI: 10.3390/v12091000.

Le Poder, S. (2011). Feline and Canine Coronaviruses: Common Genetic and Pathobiological Features. Advances in Virology. 2011, 1-11. DOI:10.1155/2011/609465.

O’Brien, A., Mettelman, R.C., Volk, A., André, N.M., Whittaker, G.R., \& Baker, S.C. (2018). Characterizing replication kinetics and plaque production of type I feline infectious peritonitis virus in three feline cell lines. Virology. 525, 1-9. DOI: 10.1016/j.virol.2018.08.022.

Pedersen, N.C. (2014). An update on feline infectious peritonitis: virology and immunopathogenesis. The Veterinary Journal. 201(2), 123-132. DOI: 10.1016/j.tvj1.2014.04.017.

Pedersen, N.C., Allen, C.E., \& Lyons, L.A. (2008). Pathogenesis of feline enteric coronavirus infection. Journal of Feline Medicine and Surgery. 10(6), 529-541. DOI: 10.1016/j.jfms.2008.02.006.

Poon, L.L.M., Chu, D.K.W., Chan, K.H., Wong, O.K., Ellis, T.M., Leung, Y.H.C., ... Peiris, J.S.M. (2005). Identification of a Novel Coronavirus in Bats. Journal of Virology. 79(4), 2001-2009. DOI: 10.1128/ jvi.79.4.2001-2009.2005.

Pratelli, A. (2008). Comparison of Serologic Techniques for the Detection of Antibodies against Feline Coronaviruses. Journal of Veterinary Diagnostic Investigation. 20(1), 45-50. DOI: 10.1177/104063870802000108.

Sharif, S., Arshad, S.S., Hair-Bejo, M., Omar, A.R., Zeenathul, N.A., \& Hafidz, M.A. (2009). Prevalence of feline coronavirus in two cat populations in Malaysia. Journal of Feline Medicine and Surgery. 11(12), 1031-1034. DOI: 10.1016/j.jfms.2009.08.005.

Su, S., Wong, G., Shi, W., Liu, J., Lai, A.C.K., Zhou, J., ... Gao, G.F. (2016). Epidemiology, Genetic Recombination, and Pathogenesis of Coronaviruses. Trends in Microbiology. 24(6), 490-502. DOI: 10.1016/j.tim.2016.03.003.

Tekes, G., \& Thiel, H.J. (2016). Feline Coronaviruses: Pathogenesis of Feline Infectious Peritonitis. Advances in Virus Research. 96, 193-218. DOI: 10.1016/bs.aivir.2016.08.002.

Worthing, K.A., Wigney, D.I., Dhand, N.K., Fawcett, A., McDonagh, P., Malik, R., \& Norris, J.M. (2012). Risk factors for feline infectious peritonitis in Australian cats. Journal of Feline Medicine and Surgery. 14(6), 405-412. DOI: 10.1177/1098612x12441875. 